\title{
Extended passaging increases the efficiency of neural differentiation from induced pluripotent stem cells
}

\author{
Karl R Koehler ${ }^{1,2}$, Philippe Tropel ${ }^{5}$, Jonathan W Theile ${ }^{1,3}$, Takako Kondo ${ }^{1,2}$, Theodore R Cummins ${ }^{1,3}$, \\ Stéphane Viville $e^{4,5}$ and Eri Hashino 1,2,3*
}

\begin{abstract}
Background: The use of induced pluripotent stem cells (iPSCs) for the functional replacement of damaged neurons and in vitro disease modeling is of great clinical relevance. Unfortunately, the capacity of iPSC lines to differentiate into neurons is highly variable, prompting the need for a reliable means of assessing the differentiation capacity of newly derived iPSC cell lines. Extended passaging is emerging as a method of ensuring faithful reprogramming. We adapted an established and efficient embryonic stem cell (ESC) neural induction protocol to test whether iPSCS (1) have the competence to give rise to functional neurons with similar efficiency as ESCS and (2) whether the extent of neural differentiation could be altered or enhanced by increased passaging.

Results: Our gene expression and morphological analyses revealed that neural conversion was temporally delayed in IPSC lines and some IPSC lines did not properly form embryoid bodies during the first stage of differentiation. Notably, these deficits were corrected by continual passaging in an IPSC clone. IPSCS with greater than 20 passages (late-passage iPSCs) expressed higher expression levels of pluripotency markers and formed larger embryoid bodies than iPSCs with fewer than 10 passages (early-passage iPSCs). Moreover, late-passage iPSCs started to express neural marker genes sooner than early-passage iPSCs after the initiation of neural induction. Furthermore, latepassage iPSC-derived neurons exhibited notably greater excitability and larger voltage-gated currents than earlypassage iPSC-derived neurons, although these cells were morphologically indistinguishable.
\end{abstract}

Conclusions: These findings strongly suggest that the efficiency neuronal conversion depends on the complete reprogramming of iPSCs via extensive passaging.

\section{Background}

Induced pluripotent stem cells (iPSCs) are somatic cells that have been epigenetically reprogrammed to a pluripotent state using the ectopic expression of defined factors (Oct3/4, Sox2, Klf4, c-myc, Nanog or Lin28) or small molecule treatments [1-5]. Like embryonic stem cells (ESCs), iPSCs have the ability to differentiate into all three germ layers and thus, represent a viable option for autologous cell replacement therapies. A number of groups have investigated the potential of iPSCs for generating in vitro models of neurodegenerative maladies, such as, Parkinson's disease, retinal degeneration, amyotrophic

\footnotetext{
* Correspondence: ehashino@iupui.edu

${ }^{1}$ Stark Neurosciences Research Institute

Full list of author information is available at the end of the article
}

lateral sclerosis and Rett Syndrome [6-14]. Although these studies are encouraging, little is currently known about the molecular underpinnings of reprogramming and the faithfulness with which iPSCs can recapitulate neuronal differentiation.

Although iPSCs of both mouse and human origins appear morphologically indistinguishable from ESCs, several reports have emerged showing variations at the transcriptomic and epigenomic levels [15-22]. In contrast, studies by Guenther et al. [23] and Neumann and Cooper [24], have shown convincingly that the discrepancies between iPSCs and ESCs are not significantly different from variations between ESC lines with divergent genetic backgrounds [23]. Moreover, laboratory-specific factors such as culture conditions and reprogramming methods may be an underlying cause of these observed

\section{C) Biomed Central}


differences [24]. Variations in teratoma forming ability, hematopoiesis and neuronal differentiation have been observed among mouse and human iPSC lines [25]. Recently, Polo et al. [26], Kim et al. [27] and Marchetto et al. [28], observed that many early-passage mouse iPSC lines maintain a persistent epigenetic signature of the tissue type of origin. Interestingly, when directed to differentiate to hematopoietic or osteogenic cell types, these early-passage cells were biased toward their original cell state, thus leading to low differentiation efficiency $[26,27]$. At later passages, the iPSCs differentiated more efficiently, which led the researchers to conclude that a period of prolonged cellular proliferation may be a necessary component of the reprogramming process. In light of these findings, it has become clear that newly derived iPSC lines should be thoroughly characterized based on their expression of endogenous pluripotency genes, morphology and differentiation capacity. However, information is lacking whether extensive passaging has effects on the competence of iPSCs to give rise efficiently to a neuronal lineage.

The goal of this study was to assess the effects of passaging on genetic stability in iPSCs and their efficiency in giving rise to functional neurons. We also wished to compare the neural differentiation potential of iPSCs with that of ESCs, by performing quantitative evaluation of temporal expression patterns of a battery of genes expressed sequentially during neural development. Due to the reported similarities between iPSC and ESCs, we hypothesized that both cells undergo similar transitions in the expression of key markers of neural differentiation. We found that iPSC lines we generated had variable competence to generate neural cells. We speculated that these discrepancies could stem from the inherent heterogeneity of iPSC cultures prior to differentiation or a residual epigenetic signature from the tissue of origin $[26,27]$. We found that, after continual passaging, an iPSC line with a low efficiency of neural conversion could recapitulate the gene expression patterns seen in ESCs undergoing neural differentiation. These findings highlight the importance of extensive cellular turnover for establishing a fully reprogrammed state in iPSCs prior to directed neural differentiation.

\section{Results \\ Newly derived mouse iPSCs show variable neural inductive ability at early-passages}

We used 4 newly established mouse iPSC lines (denoted as GG3.1/3 and miPS-20/25) and an established ESC line derived from the inner cell mass of an R1 mouse embryo (Additional file 1, Table S1) [29]. Three of the iPSC lines were generated via retroviral transduction of mouse embryonic fibroblasts with mouse Oct4, Sox2, Klf4 (miPS-20) and Nanog (miPS-25), whereas the GG3 clones were transduced with human Oct4, Sox2 and Klf4. Notably, the reprogramming factor $c-m y c$ was omitted to minimize the number of transgenes. The miPS-20/25 lines were generated using fibroblasts from transgenic mice carrying a green florescent protein (GFP) gene driven by the Oct4 promoter; therefore, pluripotency and differentiation could be monitored by the expression of GFP (Figure 1B and Additional file 1, Fig. S1A) [30]. iPSCs and ESCs were maintained and subjected to a 2step neural induction protocol (Figure 1A) as previously described [29]. All cell lines maintained a stereotypical ESC morphology (e.g. enlarged nucleus with prominent nucleoli and rounded cell clusters) in the presence of Leukemia inhibitory factor (LIF) and fetal bovine serum (Figure 1 and Additional file 1, Fig. S1). After adaptation to feeder-free conditions iPSC cultures displayed spontaneous differentiation at the edges of most cell clusters (Figure 1B-D and Additional file 1, Fig. S1). By contrast, spontaneous differentiation in ESC cultures was undetectable.

Pluripotent cells located in the center of these clusters were revealed by alkaline phosphatase staining (Additional file 1, Fig. S2A), which was consistent with GFP expression in miPS-20/25 (Figure 1B and Additional file 1, Fig. S1A). Upon dissociation and placement in serumfree cellular suspension, all cell lines formed embryoid bodies (EB), although the abundance of EBs varied greatly in iPSC cultures (data not shown). When plated and treated with neural induction medium, both ESC and iPSCs displayed characteristic neuronal epithelial morphology within 3 days (i.e. neural tube-like rosettes, Figure 1 and Additional file 1, Fig. S1; Ni3). Neurite-like processes extended from the cell clusters as early as 3 days after the start of neural induction (Figure 1B-D and Additional file 1, Fig. S1). By day 7, neuron-like cells with characteristic bipolar, multipolar and pyramidal morphologies were observed in both ESC and iPSC cultures (Figure 1B-D and Additional file 1, Fig. S1; Ni7). The prevalence of EBs with at least some non-neuronal morphologies was greater than $90 \%$ in all early-passage iPSC cultures $(n=3)$. Specifically, rhythmically beating cells with morphology resembling cardiomyocytes were observed in approximately $10 \%$ of plated iPSC EBs and multi-lineage cells were ubiquitous (Additional file 1, Fig. S1C-E, $\mathrm{n}=3$ ).

Originally, we had concerns that transgene re-expression may be a confounding factor during the differentiation process due to previous reports of this phenomenon in iPSCs derived using retroviruses [3,4]. However, analysis of endogenous transcripts for the reprogramming factors, Oct4, Sox 2 and Klf4, discounted transgene expression in the GG3.1 line (Additional file 1, Fig. S2B). The overall quality of this cell line was further ensured by expression analyses of genes in the Dlk1-Dio3 locus 


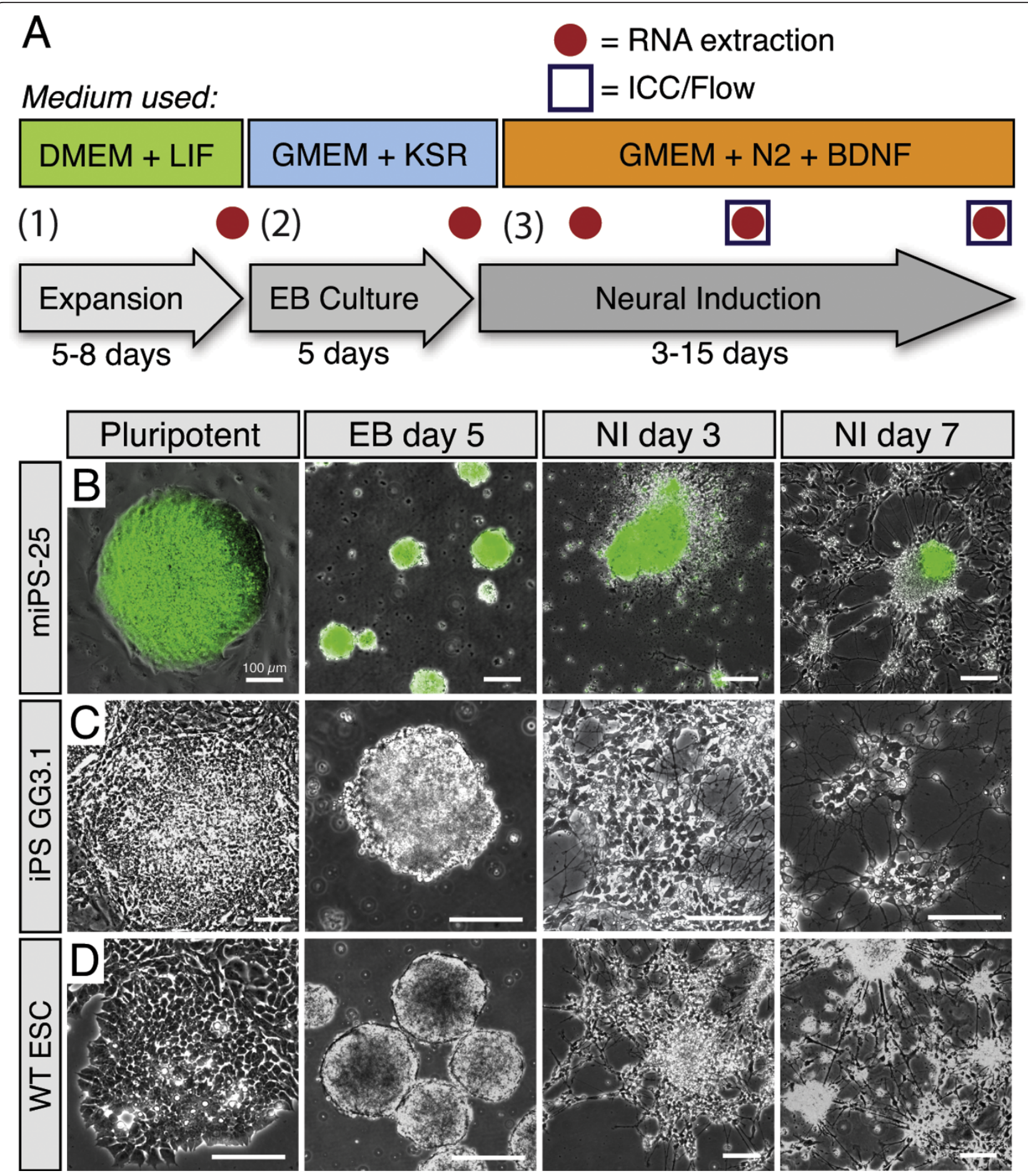

Figure 1 iPSC lines subjected to neural induction (Ni) produce populations of neurons with similar morphology to ESC-derived neurons. (A) Ni proceeds in three stages: (1) expansion of undifferentiated cells, (2) formation of embryoid bodies (EB) in a floating serum-free culture and (3) neuronal differentiation on poly-d-lysine/laminin coated plates in the presence of N2-supplement and brain derived neurotrophic factor (BDNF). (B, C) Representative micrographs for the miPS-25 (B), GG3.1 (C) and wild-type (WT) ESC lines at critical time points of neural induction: Undifferentiated, EB day 5 and Ni days 3 and 7. Bars represent $100 \mu \mathrm{m}$. 
on chromosome 12 (Additional file 1, Fig. S2C and D). Recent reports concluded that repression of this locus, specifically the genes Gtl2 and Rian, is a defining feature of poor quality mouse iPSCs that lack the ability to generate "all-iPSC mice" via tetraploid complementation $[31,32]$. We analyzed the expression level of Gtl2 and Rian in the GG3.1 line and found no difference in their expression levels when compared to ESCs (Additional file 1, Fig. S2C and D). Moreover, no significant difference in expression levels of Gtl2 and Rian was observed between early- and late-passage iPSCs (Data not shown). Considering the final differentiation performance of the GG3.1 line (i.e. post-extended passaging), this method of iPSC quality assessment should prove useful in future experiments where new iPSCs are derived.

To better characterize cellular phenotype, we performed immunocytochemistry on GG3.1 cells at neural induction day 7 . Thirty to forty percent $(n=3)$ of cells stained positive for the early neural marker $\mathrm{HuC} / \mathrm{D}$, as well as, the mature neural markers Synaptophysin (Syn), BIII-tubulin ( $\mathrm{TuJ} 1)$, microtubule associated protein 2 (MAP2) and neural nuclei protein (NeuN). As shown in previous studies, a subset of cells expressed brain-specific homeobox/POU domain protein 3A (Brn3a), indicating the presence of sensory-like neurons (Figure 2A-D). The majority of these cells were also positive for neurofilament and calretinin, consistent with our previous analysis of ESC-derived neurons (Additional file 1, Fig. S3D-F) [29]. Furthermore, we found that Map2, TuJ1, NeuN and neurofilament expression persisted beyond day 15 in iPSC cultures (data not shown). The presence of $\mathrm{Syn}^{+}$puncta and growth cones was indicative of maturing neurons (Figure 2D). This staining profile is consistent with the forebrain-like neurons observed in our and others' previous ESC analysis (See Additional file 1, Fig. S3A-F for further characterization) [29,33]. From this point on, the GG3.1 and miPS-25 lines were chosen for further analysis based on their disparate methods of generation and ability to form spherical EBs with similar abundance $\left(\sim 0.7-1 \times 10^{3} / \mathrm{mL}, \mathrm{n}=3\right)$ as ESCs.

\section{Extended passaging enhances pluripotent gene expression in an undifferentiated state and increases the rate/efficiency of neuronal conversion}

Although iPSCs exhibit neural phenotypes similar to ESCs at early-passages, we postulated that the observed morphological and differentiation inconsistencies were a result of either incomplete reprogramming or the heterogeneity of our iPSC cultures. Recent literature suggests that a prolonged period of proliferation and self-renewal may be necessary to stabilize iPSCs in a pluripotent state $[17,26]$. Accordingly, we passaged iPSCs at least 10 times prior to repetition of neural induction [26]. At 20-30 passages, spontaneous differentiation was undetectable in both GG3.1 and miPS-25 cell lines, whereas GFP expression was uniform in the miPS-25 line (Figure 3A). Interestingly, we observed a significant increase in the diameter of EBs ( 90-120 $\mu \mathrm{m}$ up to $\sim 160-190 \mu \mathrm{m}, \mathrm{n}=3)$ derived from late-passage GG3.1 cells, which was equivalent to the EB size seen in ESC cultures (Figure 3B). Furthermore, relative to early-passage iPSCs, most cells in late-passage GG3.1 cultures expressed Sox2, with few observable differentiated Sox2 cells (Figure 4A and 4B). Real-time qRT-PCR revealed that expression levels of the pluripotency markers Oct4, Sox2, Rex1 and Nanog in late-passage cultures were significantly higher than those in early-passage iPSCs and were equivalent to expression levels in ESCs (Figure 4D). Notably, Nanog expression in late-passage cells remained significantly lower than in ESCs, but there was an upward trend (Figure 4D).

To assess the transcriptional changes occurring in iPSCs over the course of neural differentiation, we carried out additional qRT-PCR using cDNA generated from undifferentiated cells, cells at EB day 5, and neural induction days 3 (Ni3), and 7 (Ni7). To clearly delineate events of gene up- and down-regulation, we evaluated the expression of immature- and mature-neuronal markers. Expression of pluripotency markers (Rex1, Oct4 and Klf4) in iPSCs declined promptly during the EB stage and subsequent differentiation (Additional file 1, Figs. S2A and S3B). The immature-neural markers, Neurogenin1 (Ngn1), Musashi1 (Msi1), Sox1 and HuC/D are all transiently expressed during in vivo neural development and have been detected in our cultures previously $[29,34]$. As expected, the mRNA levels of these genes in ESC cultures elevated during early differentiation (Ni3), but declined as neural induction proceeded (Ni7) (Figure $5 \mathrm{~A})$. By contrast, the induction of immature-neural marker genes was delayed in early-passage iPSCs (Figure 5A). However, after 20-30 passages, temporal expression patterns and levels of immature-neural markers were not significantly different from ESCs (Figure 5A). We next evaluated the expression of mature neural markers, neuron specific enolase (NSE), Syn (Figure 5A), Calretinin and TrkB (Additional file 1, Fig. S2B). We found consistently that expression of these genes is induced by $\mathrm{Ni3}$, but increases dramatically by Ni7 in ESC cultures (Figure $5 \mathrm{~A})$. This pattern of expression was seen in early-passage iPSCs, but was not as robust. As with the other markers, late-passage iPSC-derived cultures exhibited significantly higher levels of NSE and Syn expression than early-passage iPSCs at Ni7 (Figure 5A).

To better quantify the efficiency of neural differentiation, we performed flow cytometry analysis for the neural lineage marker CD24 [35-37]. Our data revealed a lower percentage of $\mathrm{CD} 24^{+}$cells in early-passage iPSC-derived cultures ( 30\%) compared to ESC-derived 


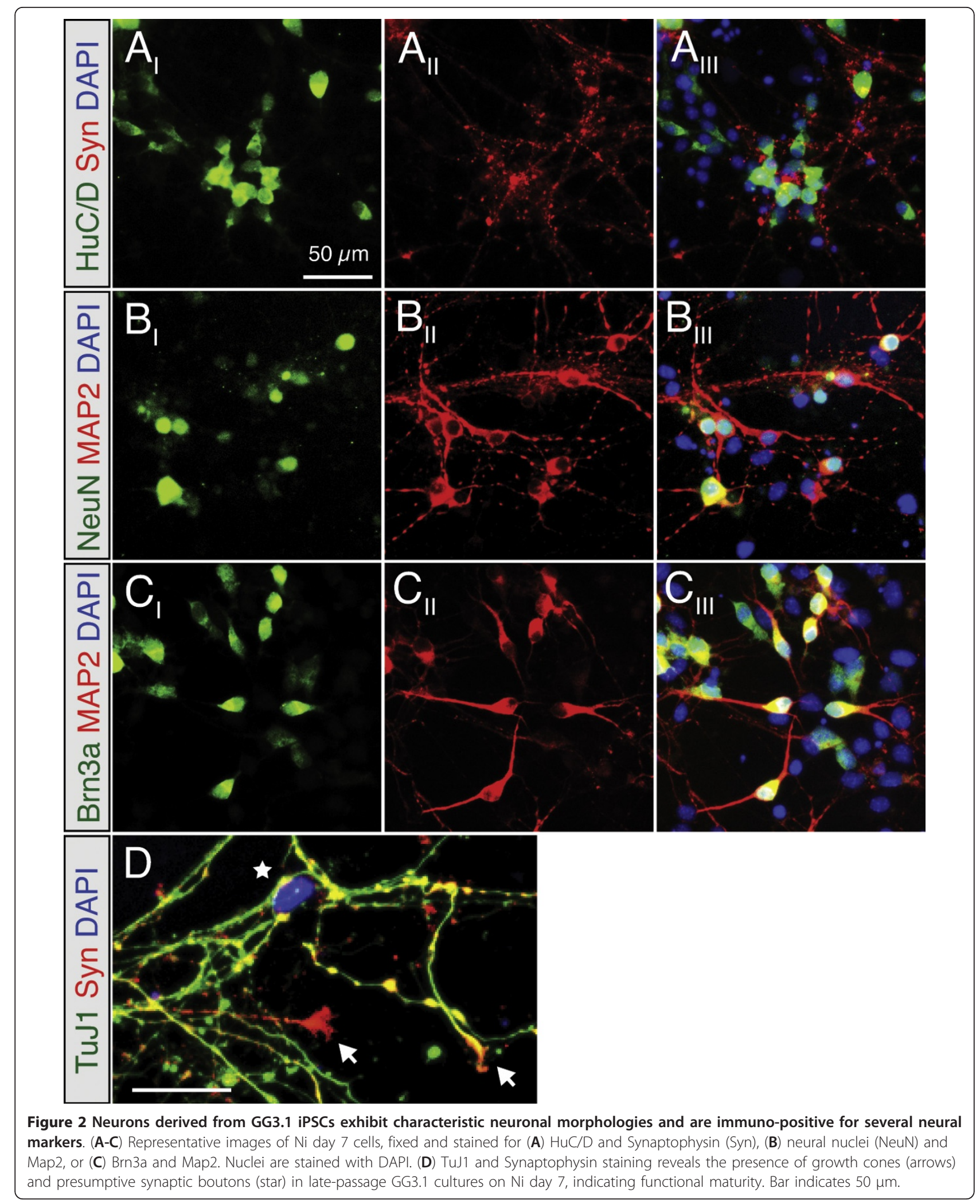




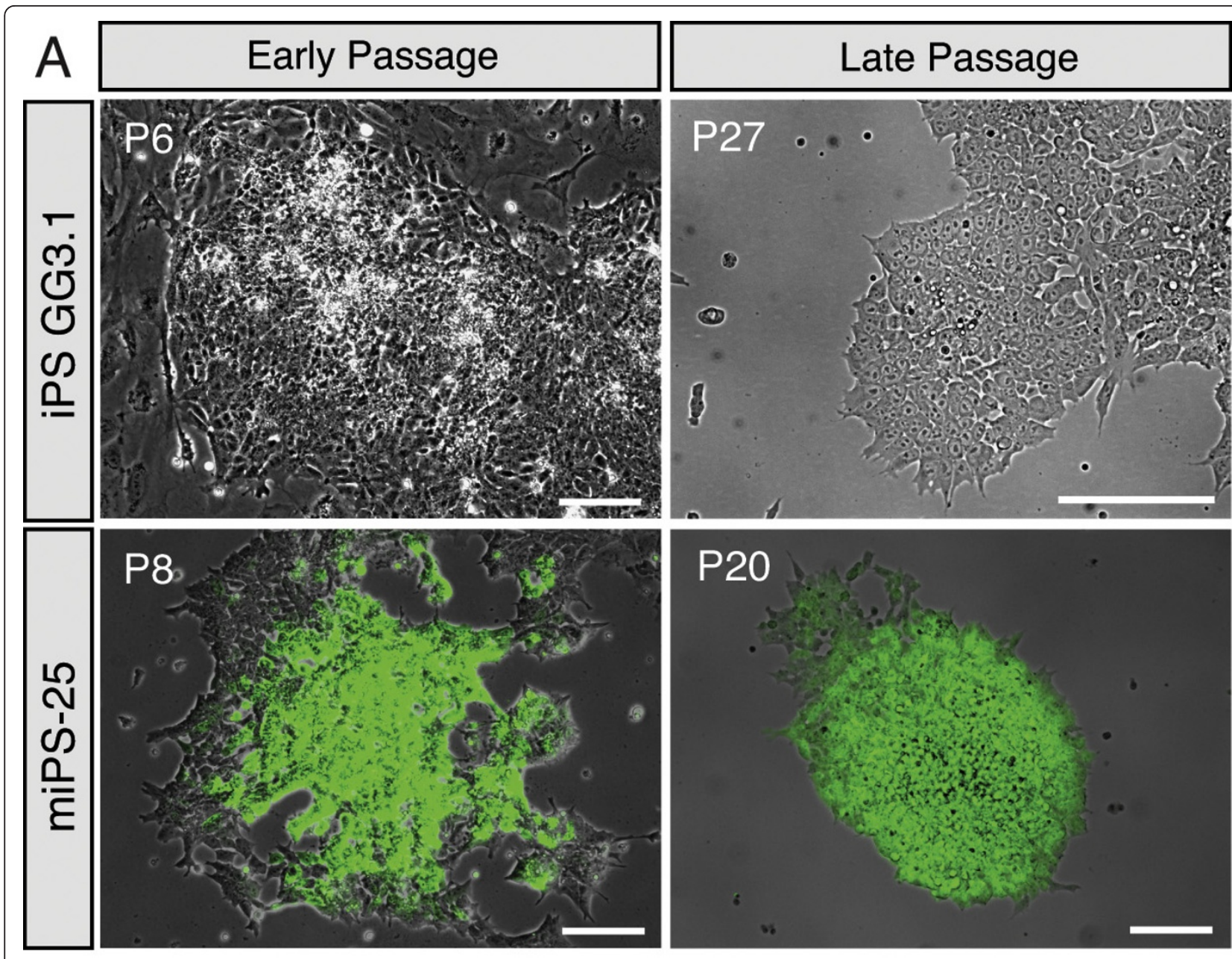

B

\section{Day 5 EB Size}

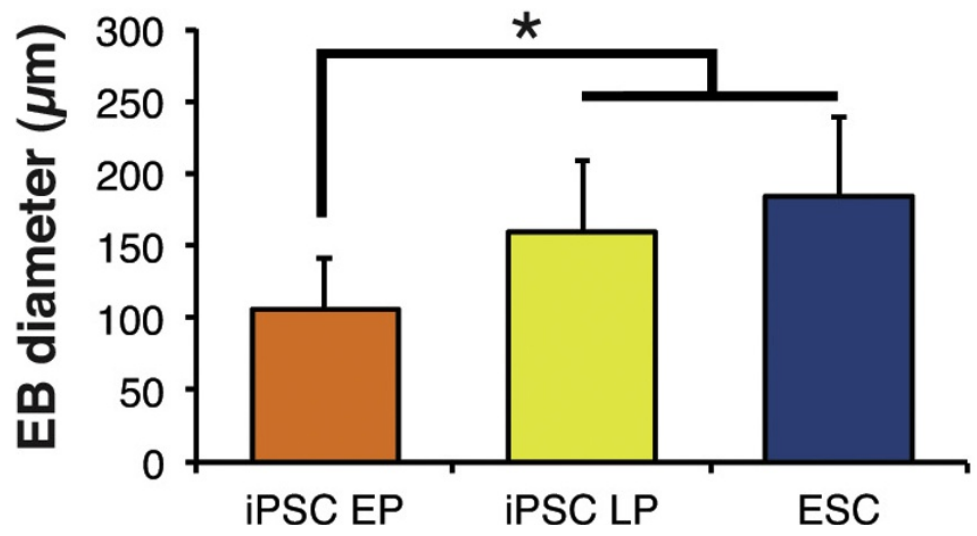

Figure 3 The effects of continual passaging on cellular morphology, colony shape and EB formation. (A) > 95\% of colonies displayed spontaneous differentiation and loss of GFP expression in peripherally located cells of low-passage GG3.1 and miPS-25 cell clusters. Serial passaging results in morphological stability and uniform GFP expression in $>85 \%$ of cell colonies in miPS-25 cultures. (B) EB diameter increased after multiple passages (iPSC LP represents cells at P20-30) compared to early-passage cultures (iPSC EP represents cells at P7-9). ESC-derived EB diameter also differs significantly from EP-derived $E B\left(n=3\right.$ for each group). Scale bars indicate $100 \mu \mathrm{m}$. Values are mean $\pm S D$. ${ }^{*} P<0.01$. 


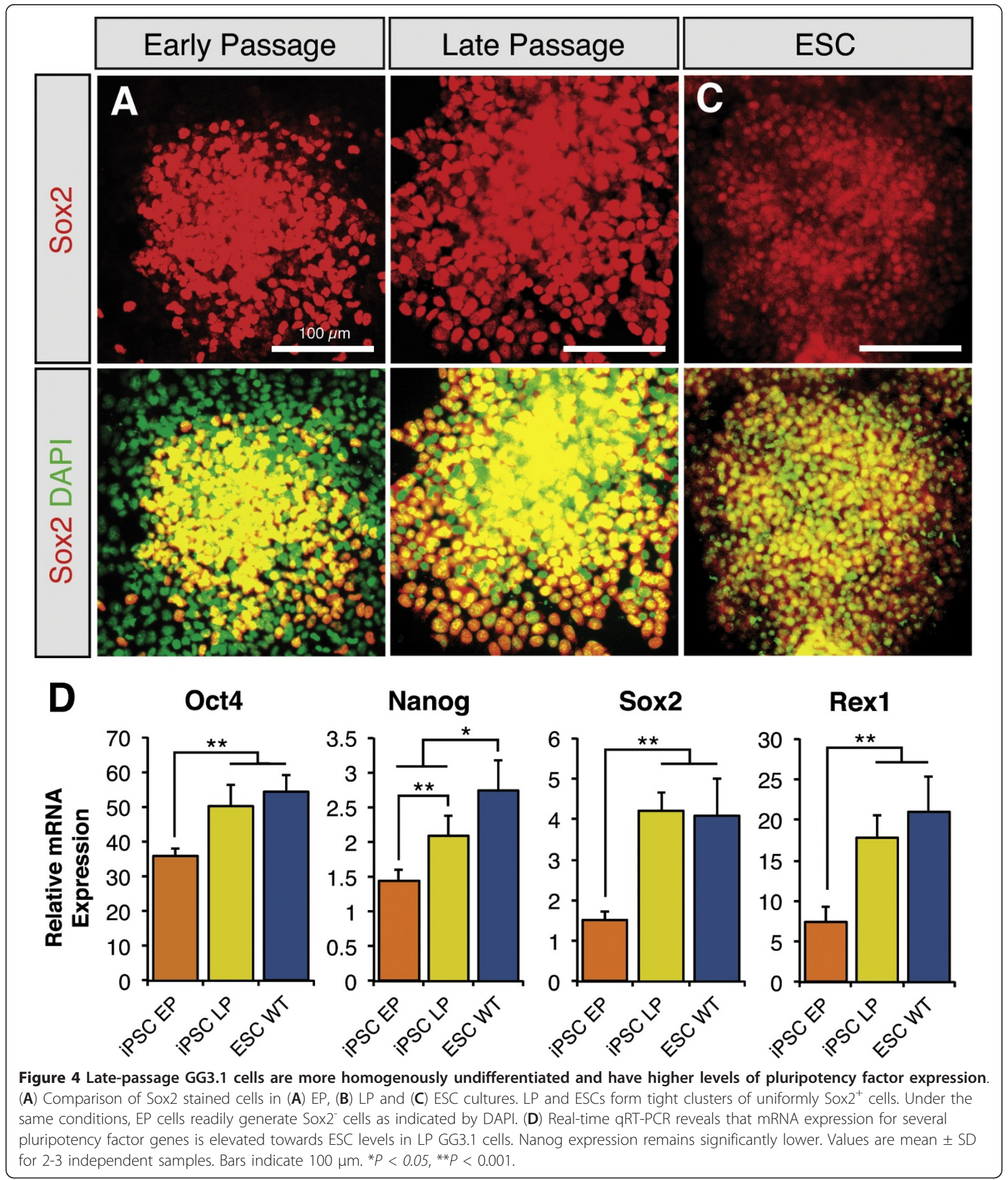

cultures $(\sim 85.5 \%)$, which was in accordance with our immunocytochemistry observations (Figure 5B). This percentage increased to approximately $50 \%$ in early-passage iPSC neural induction day 15 cultures (data not shown). Consistent with the PCR analysis, the late- passage iPSCs at neural induction day 7 contained a comparable percentage of $\mathrm{CD} 24^{+}$cells when compared to ESCs $(\sim 83 \%$, Figure $5 \mathrm{~B})$. Together, these results showed that extended passaging enhances iPSC homogeneity and similarity to ESCs in our culture system. 


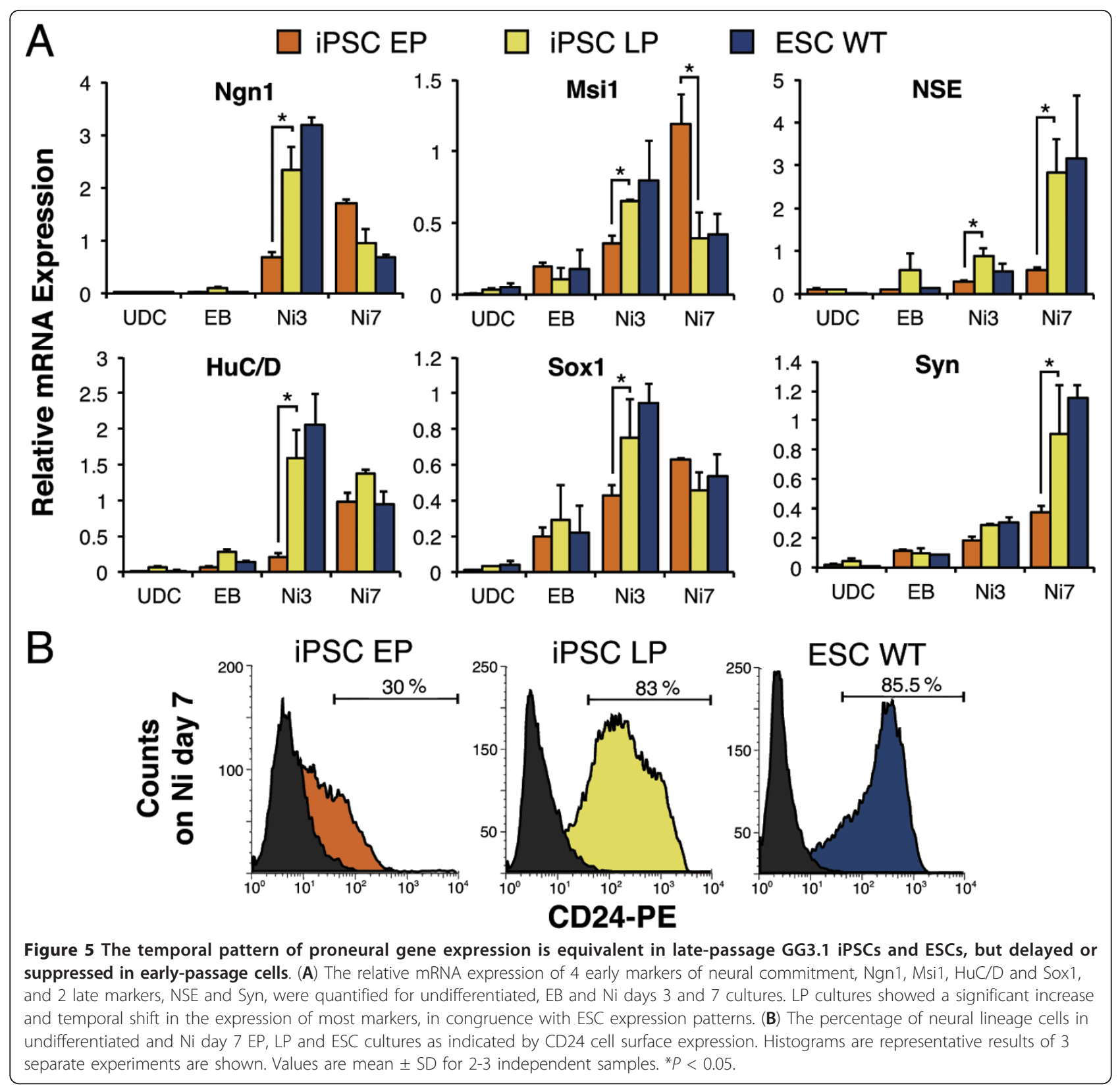

iPSC derived neurons exhibit an improved functional profile after extended passaging

To evaluate the functional status of iPSC-derived neurons, we performed whole cell patch clamp experiments between days 7-14 of neural induction (Figure 6). For consistent analysis, we chose cells with a distinct bipolar or multipolar morphology (Figure 6A). The average resting membrane potentials were similar between early and late-passage iPSCs at $\sim 55 \mathrm{mV}$, which was more depolarized than those recorded in ESCs (Figure 6B). Using a current step protocol, $~ 90 \%$ of patched ESC-derived neurons elicited repeated action potentials and robust inward and outward currents (Figure 6C). By contrast, early- passage iPSC-derived neurons, although morphologically similar to ESC-derived cells, produced only solitary or paired action potentials with comparatively weak inward and outward currents (Figure 6C). Action potentials were recorded from only $\sim 23 \%$ of cells. Hyperpolarizing the cells (to $\sim-70 \mathrm{mV}$ ) typically did not substantially enhance the ability of early-passage iPSC-derived neurons to generate repetitive action potentials. Moreover, these cells displayed poor membrane integrity, as indicated by low input resistances that tended to get even lower fairly rapidly, which made recording difficult. Late-passage iPSC-derived neurons were capable of producing action potentials of similar amplitude and frequency as 


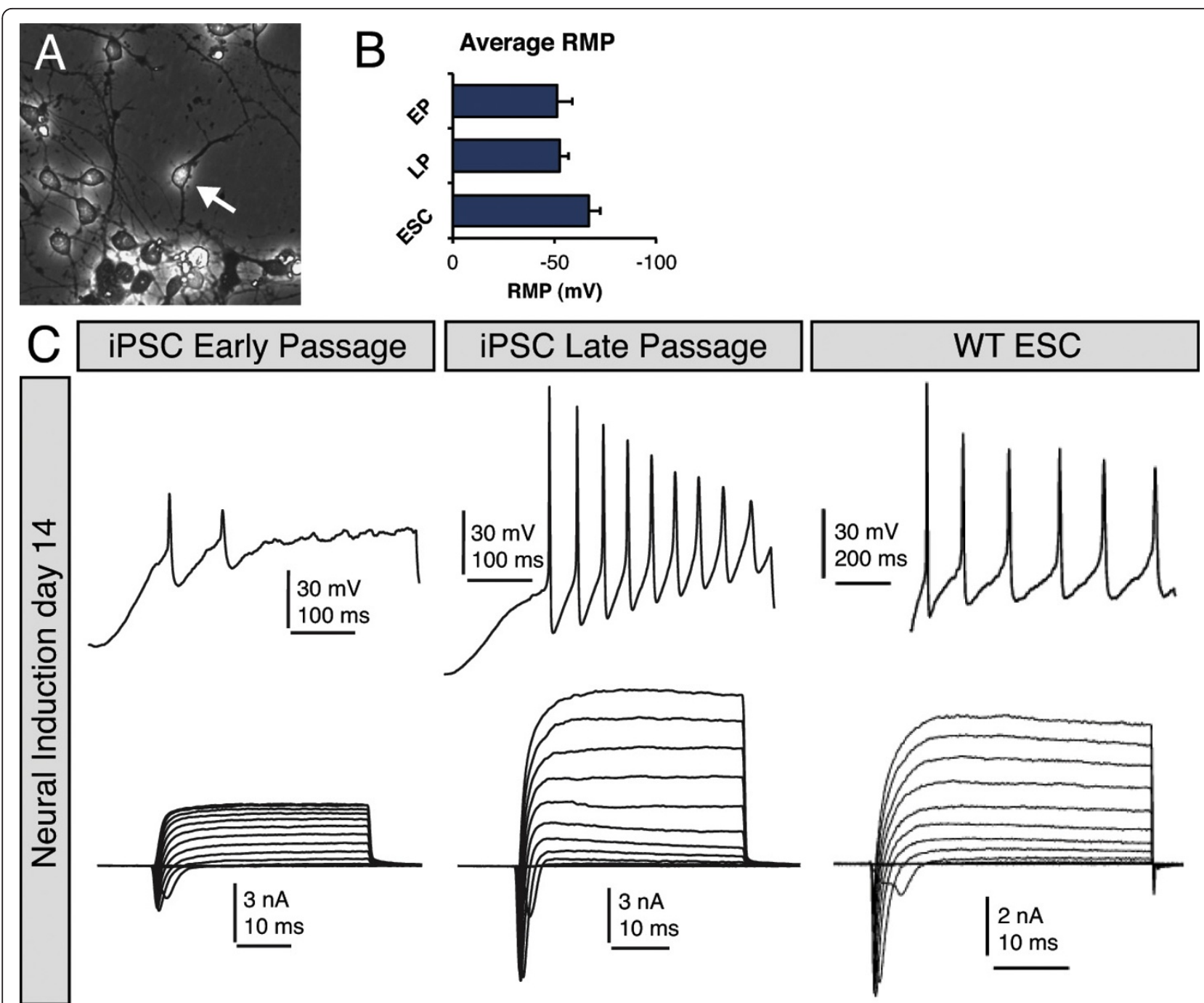

Figure 6 Electrophysiological assessment of GG3.1 iPSC-derived neurons. (A) Micrograph showing a representative neuronal cell that was targeted for recording. (B) Average resting membrane potentials for one set of experiments shows no discernable difference between EP and LP neurons. (C) Representative traces for whole cell-patch clamp recordings in EP, LP and WT ESCs. Action potentials were elicited with $500 \mathrm{~ms}$ long current injections of 2-340 pA. Current recordings were generated by stepping up membrane potential from -90 mV to +60 mV in $10 \mathrm{mV}$ increments.

ESC-derived neurons. Robust action potentials were recorded from $\sim 58 \%$ of cells (Figure $6 \mathrm{C}$ ). Accordingly, the inward and outward currents (most likely sodium and potassium currents, respectively, although this was not empirically determined) were equivalent with those detected in ESC neurons (Figure 6C).

\section{Discussion}

To our knowledge, this is the first study to specifically compare the neural differentiation capacity between early- and late-passage murine iPSCs. Of our four iPSC lines, three (GG3.1/3 and miPS-25) generated neuronal populations greater than $30 \%(n=3$ per line) of the total cell populations in early-passage culture when we applied an ESC-based neuronal induction protocol. Our group and others have previously shown that this protocol yields neuronal population of greater than $80 \%$ purity using murine ESCs $[29,33]$. Quantitative gene expression analysis revealed a similar, but temporally delayed pattern of neural lineage gene expression between ESCs and one iPSC line (GG3.1). We found that serial passaging improved the stability and maintenance of two newly derived iPSC lines in an undifferentiated state (Figure 3). Moreover, upon neural induction, late-passage iPSCs and ESCs undergo nearly identical temporal changes in gene expression (Figures 4 and 5). These results strongly suggest that sufficient cellular divisions are necessary to generated stable iPSCs clones 
that can achieve directed differentiation efficiencies comparable to ESCs.

The increase in expression of pluripotency factors in late-passage GG3.1 cells (Figure 4B) seems to agree with previous reports showing that differential gene expression between ESCs and iPSCs diminishes after passaging $[16,17]$. Since the RNAs for our analyses were extracted from whole cell populations, we must be careful in drawing conclusions about the individual cells within iPSC populations. The qRT-PCR data in Figure 4B is more an indication of the homogeneity of undifferentiated cultures, than a direct measure of pluripotency. For instance, the mRNA from early-passage cultures may be diluted by the mRNA of spontaneously differentiated cells, which would lower the measured relative expression of genes uniquely expressed in undifferentiated cells. Thusly, these data suggest that late-passage GG3.1 cultures contain a pluripotent population of cells roughly as homogeneous as our ESC cultures. Alternatively, we can conclude that the pluripotent state of these iPSC lines is more stable at later passages. Likewise, our analyses of neural markers in Figure 5 demonstrates the comparatively equivalent percentage of cells expressing these genes in late-passage GG3.1 and ESC cultures at each time point. These similarities in gene expression are particularly noteworthy when one considers that GG3.1 iPSCs and ESCs were derived from mice with disparate genetic backgrounds (i.e. B6-CD1 and R1, respectively).

Our results also point to functional differences between early-passage and late-passage iPSC-derived neurons. However, it is important to note that the results in Figure 6 are not entirely comprehensive in their assessment of each neural induction culture. For instance, we did not label a specific subtype of neurons for analysis (e.g. glutamatergic or GABAergic neurons); thus, the neurons analyzed may have represented multiple phenotypes despite having a similar morphology. In future studies, the use of subtype-specific fluorescent reporters may allow for more precise assessment of a particular population of stem cell-derived neurons. Regardless of these technical limitation, the generation of repeated action potentials with corresponding $\mathrm{Na}^{+} / \mathrm{K}^{+}$ currents was used as a general criterion for excitatory functional neurons. In early-passage cultures, we were unable to record repeated action potentials even after 14 days of differentiation. This indicates that neurons developing in early-passage cultures may be functionally defective. We speculate that the extreme heterogeneity of early-passage neural cultures may create an environment that is not conducive to functional maturation.

A growing body of work has demonstrated that iPSCs can give rise to a wide array of neural subtypes using protocols optimized for ESCs $[9,12,38,39]$. However, few studies consider thoroughly the relative efficiency with which differentiation occurs between ESCs and iPSCs. Recently, $\mathrm{Hu}$ et al. published work showing that human iPSC lines derived using disparate methods (i.e. integrating and non-integrating vectors) displayed variable efficiencies when directed to differentiate into motor neurons [38]. Remarkably, cell lines derived using nonintegrating episomal expression of the transgenes appeared to be just as susceptible to variation in differentiation potency as cells derived using retroviruses, which suggests that variability is independent of derivation method. These findings are reminiscent of our initial comparison of early-passage iPSCs and ESCs in that differentiation potency failed to match that seen in ESCs. It is noteworthy that the passage numbers of the iPSC cell lines used by $\mathrm{Hu}$ et al. were not reported, so it is possible that these observed differences could be attenuated with sufficient cellular turnover. More recently, Boulting et al. found that early- and late-passage human iPSCs performed similarly during motor neuron differentiation and functional analysis, despite karyotypic abnormalities in some late-passage cell lines [39].

Since varying differentiation propensities among iPSC lines appear to be independent of derivation methods, the beneficial effect of repeated passaging may reveal an underlying feature of cellular reprogramming in general. It has been proposed that a residual signature or "memory" of the cell type of origin persists throughout the reprogramming process in the form of hypo- or hypermethylated regions of the genome and/or aberrant gene expression [26-28]. It is possible that hypermethylation of neural gene promoter regions may have confounded early-passage iPSC differentiation, although we did not directly test this. Several new studies also report the generation of genetic mutations, deletions and copy number variations during the reprogramming process $[18,19,21]$. Over successive cellular divisions, however, it appears that epigenetic marks are progressively "erased" or, perhaps, selected against. At the moment, the precise mechanisms of this process are unclear, but the epigenetic signature appears to be a phenomenon in both mouse and human reprogrammed cells [17,26,27]. Of note, Hussein and colleagues recently demonstrated that early-passage human iPSC lines have a high prevalence of genetic copy number variations. Surprisingly, the amount of copy number variations declined rapidly over successive passages (i.e. > 15 passages) seemingly due to selective pressure on the aberrant cells [19]. It is feasible that this phenomenon is reflected in our current observations. For future investigations it will be necessary to examine karyotypic stability and copy number variation over the course of these experiments to determine if neural differentiation is impacted by these factors. 


\section{Conclusions}

The work presented herein demonstrates that extended passaging can lead to more stable iPSCs, which in turn leads to more efficient neural differentiation. The utility of this approach will certainly be elucidated by further studies examining the effect of passaging on chromosomal stability in iPSCs. Importantly, the present results highlight the need for improved screening methodologies to isolate iPSC clones with the greatest potential for directed differentiation. Future studies identifying methylation signatures that define fully reprogrammed iPSCs will be helpful in developing better assays to evaluate the progression of reprogramming. Interestingly, some reports suggest that neuronal conversion of recalcitrant iPSCs can be greatly improved through treatment with chromatin-modifying drugs or small molecules $[27,39,40]$. Undoubtedly, for the eventual application of iPSCs in disease modeling or cell replacement therapies, complete reprogramming will be critical for unbiased analysis of disease progression and safety.

\section{Methods}

\section{ES and iPS cell culture, maintenance and analysis}

iPSCs were generated by transducing mouse embryonic fibroblasts (for genetic backgrounds see Supplementary Table 1 in Additional File 1) with Moloney murine leukemia viruses (MMLVs) carrying the coding regions of mouse Oct4, Sox2, Klf4 and/or Nanog or human Oct4, Sox 2 and Klf4. R1 mouse embryonic stem cells and iPSCs were maintained in culture as described previously (Figure 1A) [29]. Briefly, iPS and ES cells were plated on gelatin-coated tissue culture plates and grown in high-glucose Dulbecco's Modified Eagle's Medium (DMEM) (Invitrogen) supplemented with 15\% FBS (Invitrogen), $1.0 \mathrm{mM}$ sodium pyruvate (Stemcell Technologies), $10 \mathrm{mM}$ nonessential amino acids (Stemcell Technologies), $0.01 \%$ penicillin streptomycin (Stemcell Technologies), $2.0 \mathrm{mM}$ L-glutamine (Stemcell Technologies), 1,000 units/ml leukemia inhibiting factor (Chemicon), and $0.055 \mathrm{mM} 2$-mercaptoethanol. Cells were passaged by dissociation with $0.25 \%$ trypsin-EDTA every 2-3 days. Two days after passaging the health and phenotypic stability of the cells was assessed. Five to ten representative DIC images were taken and then analyzed on MetaMorph software. Dissociation of tightly packed clones and/or the appearance of enlarged and flattened cells were indicators of spontaneous differentiation.

\section{Neural induction}

After 6-8 (early) and 20-30 (late) passages, iPSC and latepassage (30-40) ESCs were subjected to neural differentiation according to a previously established procedure for ESCs (Figure 1A) [29,33]. Cells were dissociated into single cells using $0.25 \%$ trypsin-EDTA and resuspended in differentiation medium containing Glasgow's Minimum Essential Medium (GMEM) (GIBCO/Invitrogen), $5 \%$ Knockout serum replacement (Invitrogen), $2.0 \mathrm{mM}$ L-glutamine, $1.0 \mathrm{mM}$ sodium pyruvate, $0.1 \mathrm{mM}$ nonessential amino acids, $0.01 \%$ penicillin streptomycin, and $0.1 \mathrm{mM} 2$-mercaptoethanol. Cells were plated on gelatincoated plates for 40 minutes to remove any residual feeder cells or partially differentiated cells. Cells were then cultured in low adherence $100 \mathrm{~mm}$ bacterial plates for 5 days at a density of $5-10 \times 10^{4}$ (iPSC) or $5 \times 10^{4}$ (ESC) cells per $\mathrm{ml}$ to allow embryoid body (EB) formation. Differentiation medium was changed at day 3 . On day 5 , EBs were plated en bloc on tissue culture plates or chamber slides double-coated with poly-D-lysine $(200 \mu \mathrm{g} / \mathrm{ml})$ and mouse laminin $(10 \mu \mathrm{g} / \mathrm{ml})$ at a density of $1-2 \times 10^{2}$ EBs per $\mathrm{cm}^{2}$ in fresh medium. Before plating, EB were imaged to assess size and shape. At least $50 \mathrm{EBs}$ were analyzed using MetaMorph software to determine the average EB diameter for each biological replicate. Twenty-four-thirty-six hours post plating, the medium was changed to neural induction medium containing GMEM, 1\% N2, $2 \mathrm{mM}$ glutamine, $1 \mathrm{mM}$ sodium pyruvate, $0.1 \mathrm{mM}$ nonessential amino acids, $0.1 \mathrm{mM} 2$ mercaptoethanol, $0.01 \%$ penicillin streptomycin and $10 \mathrm{ng} / \mathrm{ml}$ brain-derived neurotrophic factor (BDNF) (PeproTech). Neural induction cultures were maintained for 3, 7 or 15 days before cells were harvested for RNA extraction, electrophysiological recordings, flow cytometry analysis, or fixation with $4 \%$ paraformaldehyde for immunocytochemistry.

\section{Quantitative RT-PCR}

The relative expression levels of pluripotency markers and early/mature neural markers were assessed by conventional reverse transcriptase PCR (RT-PCR) or quantitative real-time RT-PCR (qRT-PCR) using a previously described procedure [41]. At various time points of cell culture and neural induction (undifferentiated day 5-7, EB day 5, and days 3, 7 and 15 of neural induction), total RNA was isolated using the RNeasy Minikit (Qiagen) and then treated with TURBO DNase (Ambion) to decrease the likelihood of DNA contamination. Single-stranded cDNA was synthesized using Omniscript reverse transcriptase (Qiagen) and Oligo-dT primers. All amplicons had standardized sizes of 100-110 bps. For non-quantitative RT-PCR, the resultant cDNA was amplified with Platinum Taq DNA polymerase (Invitrogen) for 30 cycles. For qRT-PCR, the cDNA samples were amplified on an ABI PRISM 7900HT Sequence Detection System (Applied Biosystems) using the SYBR Green PCR Master Mix (Applied Biosystems). For each PCR reaction, a mixture containing cDNA template (5 $\mathrm{ng})$, Master Mix, and 
forward and reverse primers (400 $\mathrm{nM}$ each) was treated with uracil $\mathrm{N}$-glycosylase at $50^{\circ} \mathrm{C}$ for 2 min before undergoing the following program: 1 cycles, $95^{\circ} \mathrm{C}, 10 \mathrm{~min} ; 45$ cycles, $95^{\circ} \mathrm{C}, 15 \mathrm{sec}, 60^{\circ} \mathrm{C}, 1 \mathrm{~min} ; 1$ cycles, $95^{\circ} \mathrm{C}, 15 \mathrm{sec}$, $60^{\circ} \mathrm{C}, 15 \mathrm{sec}, 95^{\circ} \mathrm{C}, 15 \mathrm{sec}$ (for melting curve analysis); $72^{\circ} \mathrm{C}$, hold. Melting curve analysis was performed to confirm the authenticity of the PCR products. For internal control, PCR was run with cDNA samples using an L27 (ribosomal housekeeping gene) primer pair, whose PCR product crosses an intron. To check the efficiency of primer pairs, a cDNA dilution series $(1,1 / 10,1 / 100$, and $1 / 1,000)$ was amplified. The mRNA level for each gene was calculated relative to L27 mRNA expression. L27 expression was previously determined to be stable under all experimental conditions [29]. Each data point represents the average of 7-10 replicates from 3-4 biological samples. Statistical significance was determined using a One-Way ANOVA followed by Scheffe's post-hoc test. Primer sequences used in this study are listed in Supplementary Table 2 (Additional File 1).

\section{Immunocytochemistry}

Prior to differentiation and at days 3 and 7 of neural differentiation, cultures were fixed with $4 \%$ paraformaldehyde for $30 \mathrm{~min}$. Chamber slides were incubated in blocking solution and then with a primary polyclonal and a monoclonal antibody together. Primary antibodies used in this study are listed in Supplementary Table 3 (Additional File 1). Immunoreactivity with monoclonal and polyclonal antibodies was visualized by using an Alexa Fluor 488 conjugated anti-mouse IgG and Alexa Fluor 568 conjugated anti-rabbit IgG, respectively. For visualizing cellular nuclei, the specimens were counterstained with DAPI (Vector, VectaShield). Expression of certain proteins was quantified using the imageJ (NIH) cell counting plug-in. Regions with moderate cellular densities were chosen at random for 3 biological samples unless stated otherwise.

\section{Electrophysiology}

Whole cell patch-clamp recordings were conducted as described previously [29]. Briefly, experiments were performed using an EPC-10 amplifier, and data was acquired using the Pulse program (HEKA Electronics). Putative bipolar neurons were selected for recording based on morphology. The pipette solution contained: $140 \mathrm{mM} \mathrm{KCl}, 5 \mathrm{mM} \mathrm{MgCl} 2,5 \mathrm{mM}$ EGTA, $2.5 \mathrm{mM}$ $\mathrm{CaCl}$, $4 \mathrm{mM}$ ATP, $0.3 \mathrm{mM}$ GTP, and $10 \mathrm{mM}$ Hepes, pH 7.3 (adjusted with $\mathrm{KOH}$ ). The bathing solution contained: $140 \mathrm{mM} \mathrm{NaCl}, 1 \mathrm{mM} \mathrm{MgCl} 2,5 \mathrm{mM} \mathrm{KCl,} 2 \mathrm{mM}$ $\mathrm{CaCl} 2,10 \mathrm{mM}$ Hepes, and $10 \mathrm{mM}$ glucose, $\mathrm{pH} 7.3$ (adjusted with $\mathrm{NaOH}$ ). Voltage-clamp and currentclamp data was analyzed using the Pulsefit (HEKA
Electronics), Origin (OriginLab) and Microsoft Excel software.

\section{Flow cytometry}

Cells were dissociated by a brief exposure to $0.25 \%$ trypsin-EDTA. After blocking with serum, cells were incubated with one of the following primary antibodies: antiCD24-phycoerythrin (PE), mouse immunoglobulin G (IgG) isotype control or Alexa 568-conjugated anti-rabbit secondary antibody. Cell sorting and analysis were performed with a FACSCalibur flow cytometry system (BD Biosciences). Data analysis was performed using FlowJo 8.6.6 software (Tree Star, Inc.).

\section{Additional material}

Additional File 1: Supplementary information. Supplementary Figures 1-3 and Tables 1-3. Supplementary Figure 1 - iPSCs (GG3.3 and miPS20) at various stages of neural differentiation. Representative micrographs of miPS-20 (A) and GG3.3 (B) iPSCs prior to differentiation, on day 5 of EB formation and on days 3 and 7 of neural induction. (C-E) Examples of aberrant cell types with endodermal (C) and mesodermal (D, E) morphologies that were prevalent during all early-passage iPSC Ni experiments. Scale bars represent $100 \mu \mathrm{m}$. Supplementary Figure 2 The GG3.1 cell line is a competent iPSC line with no detectable transgene re-expression during neural differentiation. (A) Alkaline phosphatase staining of ESC and GG3.1 cells indicates pluripotent cells in undifferentiated cultures and a gradual loss of pluripotency during the EB stage. (B) Primers amplifying an untranslated region (UTR) of the Oct4, Sox2 and Klf4 genes were compared to exon expression in undifferentiated and neural induction days 3 and 7. All expression levels were normalized to undifferentiated expression levels. The identical pattern of expression indicates a lack of transgene re-expression. (C-D) The GG3.1 cell line displays similar expression levels of the Dlk1-Dio3 locus genes Gtl2 and Rian, which is an indirect measure of complete reprogramming. Equivalent expression of these genes was validated using 2 different primer sets; one novel and one published by Stadtfeld et al., 2010. Values are mean \pm SD for 2-3 independent samples. Supplementary Figure 3 - Expression of neural lineage and subtype specific genes throughout $\mathrm{Ni}$ of early-passage GG3.1 iPSCs. (A-C) Representative micrographs showing the presence and abundance of HuC/D, Map2, neurofilament (NF) and Calretinin (Calr) positive cells at $\mathrm{Ni}$ day 7. Scale bars represent $150 \mu \mathrm{m}$. (D) The pluripotency marker Rex1 is downregulated during differentiation. (E) The anterior

neurodevelopmental gene Otx2 is expressed by day 5 of EB. The neurotrophin receptor TrkB is expressed during the EB stage, but expression is elevated by day 7 of $\mathrm{Ni}$. Calretinin is expressed by $\mathrm{Ni}$ day 3 . (F) Markers of glutamatergic neurons, vesicular glutamate transporter 2 (VGLUT2) and the AMPA receptor subunit GluR2 are highly expressed by days 7 and 15 of Ni. Likewise, the GABAergic neuronal marker, glutamic acid decarboxylase 1 (GAD1) is unregulated by days 7 and 15. Values are mean \pm SD for 2-3 independent samples. Supplementary Table 1 Pluripotent stem cell IInes. Supplementary Table 2 - Primers. Supplementary Table 3 - Antibodies.

\section{Acknowledgements}

The authors would like to thank Elizabeth Tobin, Emily Beans and Riddhi Trivedi for their helpful comments and technical support during the preparation of this manuscript. This work was supported by the National Institutes of Health grants RC1DC010706 (to EH) and R01NS053422 (to TRC), as well as by funds provided from the French Centre National de la Recherche Scientifique (CNRS), Institut National de la Santé et de la Recherche Médicale (INSERM), the Ministère de I'Education Nationale, the 
I'Enseignement Supérieur et de la Recherche, the University of Strasbourg and l'Association Française contre les Myopathies (AFM) (to SV). KRK was supported by a Paul and Carole Stark Neurosciences Fellowship and an Indiana Clinical and Translational Science Institute Pre-doctoral Fellowship (NIH TL1RR025759), whereas JWT was supported by a PhRMA Foundation Postdoctoral Fellowship.

\section{Author details}

${ }^{1}$ Stark Neurosciences Research Institute. ${ }^{2}$ Department of Otolaryngology. ${ }^{3}$ Department of Pharmacology and Toxicology, Indiana University School of Medicine, Indianapolis, IN 46202, USA. ${ }^{4}$ Service de Biologie de la Reproduction, Centre Hospitalier Universitaire, Strasbourg, F-67000 France. ${ }^{5}$ Institut de Génétique et de Biologie Moléculaire et Cellulaire (IGBMC), Institut National de Santé et de Recherche Médicale (INSERM) U964/Centre National de Recherche Scientifique (CNRS) UMR 1704/Université de Strasbourg, 67404 Illkirch, France.

\section{Authors' contributions}

KRK designed the experiments and performed the cell culture, immunocytochemistry, flow cytometry and GPCR analysis and drafted the manuscript. PT and SV generated the iPSC lines. TK assisted with cell culture and data analysis. JWT and TRC conducted the electrophysiological analyses. $\mathrm{EH}$ aided in the conceptual design of the study, monitored the experiments and helped draft the manuscript. All authors read and approved the final manuscript.

Received: 19 April 2011 Accepted: 10 August 2011 Published: 10 August 2011

\section{References}

1. Takahashi K, Yamanaka S: Induction of pluripotent stem cells from mouse embryonic and adult fibroblast cultures by defined factors. Cell 2006, 126(4):663-676.

2. Takahashi K, Tanabe K, Ohnuki M, Narita M, Ichisaka T, Tomoda K, Yamanaka S: Induction of pluripotent stem cells from adult human fibroblasts by defined factors. Cell 2007, 131(5):861-872.

3. Park I-H, Zhao R, West JA, Yabuuchi A, Huo H, Ince TA, Lerou PH, Lensch MW, Daley GQ: Reprogramming of human somatic cells to pluripotency with defined factors. Nature 2008, 451(7175):141-146.

4. Yu J, Vodyanik MA, Smuga-Otto K, Antosiewicz-Bourget J, Frane JL, Tian S, Nie J, Jonsdottir GA, Ruotti V, Stewart R, Slukvin II, Thomson JA: Induced pluripotent stem cell lines derived from human somatic cells. Science 2007, 318(5858):1917-1920.

5. Ichida JK, Blanchard J, Lam K, Son EY, Chung JE, Egli D, Loh KM, Carter AC, Giorgio FPD, Koszka K, Huangfu D, Akutsu H, Liu DR, Rubin LL, Eggan K: A small-molecule inhibitor of tgf-Beta signaling replaces sox 2 in reprogramming by inducing nanog. Cell Stem Cell 2009, 5(5):491-503.

6. Osakada F, Ikeda H, Sasai Y, Takahashi M: Stepwise differentiation of pluripotent stem cells into retinal cells. Nat Protoc 2009, 4(6):811-824.

7. Muñoz-Sanjuán I, Brivanlou AH: Neural induction, the default model and embryonic stem cells. Nat Rev Neurosci 2002, 3(4):271-280.

8. Li M, Pevny L, Lovell-Badge R, Smith A: Generation of purified neural precursors from embryonic stem cells by lineage selection. Curr Biol 1998, 8(17):971-974.

9. Karumbayaram S, Novitch BG, Patterson M, Umbach JA, Richter L, Lindgren A, Conway AE, Clark AT, Goldman SA, Plath K, Wiedau-Pazos M, Kornblum HI, Lowry WE: Directed differentiation of human-induced pluripotent stem cells generates active motor neurons. Stem Cells 2009, 27(4):806-811.

10. Chambers SM, Fasano CA, Papapetrou EP, Tomishima M, Sadelain M, Studer L: Highly efficient neural conversion of human ES and iPS cells by dual inhibition of SMAD signaling. Nat Biotechnol 2009, 27(3):275-280.

11. Cai J, Yang M, Poremsky E, Kidd S, Schneider JS, lacovitti L: Dopaminergic Neurons Derived from Human Induced Pluripotent Stem Cells Survive and Integrate into 6-OHDA Lesioned Rats. Stem Cells Dev 2009, 19(7):1017-1023

12. Wernig M, Zhao J-P, Pruszak J, Hedlund E, Fu D, Soldner F, Broccoli V, Constantine-Paton M, Isacson $\mathrm{O}$, Jaenisch R: Neurons derived from reprogrammed fibroblasts functionally integrate into the fetal brain and improve symptoms of rats with Parkinson's disease. Proc Natl Acad SC USA 2008, 105(15):5856-5861.
13. Muotri AR, Marchetto MCN, Coufal NG, Oefner R, Yeo G, Nakashima K, Gage FH: L1 retrotransposition in neurons is modulated by $\mathrm{MeCP} 2$. Nature 2010, 468(7322):443-446.

14. Marchetto MCN, Carromeu C, Acab A, Yu D, Yeo GW, Mu Y, Chen G, Gage FH, Muotri AR: A model for neural development and treatment of rett syndrome using human induced pluripotent stem cells. Cell 2010, 143(4):527-539.

15. Doi A, Park I-H, Wen B, Murakami P, Aryee MJ, Irizarry R, Herb B, LaddAcosta C, Rho J, Loewer S, Miller J, Schlaeger T, Daley GQ, Feinberg AP: Differential methylation of tissue- and cancer-specific CpG island shores distinguishes human induced pluripotent stem cells, embryonic stem cells and fibroblasts. Nature Genetics 2009, 41(12):1350-1353.

16. Chin MH, Mason MJ, Xie W, Volinia S, Singer M, Peterson C, Ambartsumyan G, Aimiuwu O, Richter L, Zhang J, Khvorostov I, Ott V, Grunstein M, Lavon N, Benvenisty N, Croce CM, Clark AT, Baxter T, Pyle AD, Teitell MA, Pelegrini M, Plath K, Lowry WE: Induced pluripotent stem cells and embryonic stem cells are distinguished by gene expression signatures. Cell Stem Cell 2009, 5(1):111-123.

17. Chin MH, Pellegrini M, Plath K, Lowry WE: Molecular Analyses of Human Induced Pluripotent Stem Cells and Embryonic Stem Cells. Cell Stem Cell 2010, 7(2):263-269.

18. Gore A, Li Z, Fung H-L, Young JE, Agarwal S, Antosiewicz-Bourget J, Canto I, Giorgetti A, Israel MA, Kiskinis E, Lee J-H, Loh Y-H, Manos PD, Montserrat N, Panopoulos AD, Ruiz S, Wilbert ML, Yu J, Kirkness EF, Izpisua Belmonte JC, Rossi DJ, Thomson JA, Eggan K, Daley GQ, Goldstein LSB, Zhang K: Somatic coding mutations in human induced pluripotent stem cells. Nature 2011, 471(7336):63-67.

19. Hussein SM, Batada NN, Vuoristo $S$, Ching RW, Autio R, Närvä E, Ng S, Sourour M, Hämäläinen R, Olsson C, Lundin K, Mikkola M, Trokovic R, Peitz M, Brüstle O, Bazett-Jones DP, Alitalo K, Lahesmaa R, Nagy A Otonkoski T: Copy number variation and selection during reprogramming to pluripotency. Nature 2011, 471(7336):58-62

20. Laurent LC, Ulitsky I, Slavin I, Tran H, Schork A, Morey R, Lynch C, Harness JV, Lee S, Barrero MJ, Ku S, Martynova M, Semechkin R, Galat V, Gottesfeld J, Izpisua Belmonte JC, Murry C, Keirstead HS, Park H-S, Schmidt U, Laslett AL, Muller F-J, Nievergelt CM, Shamir R, Loring JF: Dynamic changes in the copy number of pluripotency and cell proliferation genes in human ESCs and iPSCs during reprogramming and time in culture. Cell Stem Cell 2011, 8(1):106-118.

21. Lister R, Pelizzola M, Kida YS, Hawkins RD, Nery JR, Hon G, AntosiewiczBourget J, O'Malley R, Castanon R, Klugman S, Downes M, Yu R, Stewart R, Ren B, Thomson JA, Evans RM, Ecker JR: Hotspots of aberrant epigenomic reprogramming in human induced pluripotent stem cells. Nature 2011, 471(7336):68-73

22. Mayshar Y, Ben-David U, Lavon N, Biancotti J-C, Yakir B, Clark AT, Plath K, Lowry WE, Benvenisty N: Identification and classification of chromosomal aberrations in human induced pluripotent stem cells. Cell Stem Cell 2010, 7(4):521-531.

23. Guenther MG, Frampton GM, Soldner F, Hockemeyer D, Mitalipova M, Jaenisch R, Young RA: Chromatin Structure and Gene Expression Programs of Human Embryonic and Induced Pluripotent Stem Cells. Cell Stem Cell 2010, 7(2):249-257.

24. Newman AM, Cooper JB: Lab-Specific Gene Expression Signatures in Pluripotent Stem Cells. Cell Stem Cell 2010, 7(2):258-262.

25. Miura K, Okada Y, Aoi T, Okada A, Takahashi K, Okita K, Nakagawa M, Koyanagi M, Tanabe K, Ohnuki M, Ogawa D, Ikeda E, Okano H, Yamanaka S: Variation in the safety of induced pluripotent stem cell lines. Nat Biotechnol 2009, 27(27):743-745

26. Polo JM, Liu S, Figueroa ME, Kulalert W, Eminli S, Tan KY, Apostolou E, Stadtfeld M, Li Y, Shioda T, Natesan S, Wagers AJ, Melnick A, Evans T, Hochedlinger K: Cell type of origin influences the molecular and functional properties of mouse induced pluripotent stem cells. Nat Biotechnol 2010, 28(28):848-855.

27. Kim K, Doi A, Wen B, Ng K, Zhao R, Cahan P, Kim J, Aryee MJ, Ji H, Ehrlich LIR, Yabuuchi A, Takeuchi A, Cunniff KC, Hongguang H, MckinneyFreeman S, Naveiras $O$, Yoon TJ, Irizarry RA, Jung N, Seita J, Hanna J, Murakami P, Jaenisch R, Weissleder R, Orkin SH, Weissman IL, Feinberg AP, Daley GQ: Epigenetic memory in induced pluripotent stem cells. Nature 2010, 467(467):285-290.

28. Marchetto MCN, Yeo GW, Kainohana O, Marsala M, Gage FH, Muotri AR: Transcriptional signature and memory retention of human-induced pluripotent stem cells. PLOS ONE 2009, 4(9):e7076. 
29. Kondo T, Sheets PL, Zopf DA, Aloor HL, Cummins TR, Chan RJ, Hashino E: TIx3 exerts context-dependent transcriptional regulation and promotes neuronal differentiation from embryonic stem cells. Proc Natl Acad Sci USA 2008, 105(15):5780-5785.

30. Yoshimizu T, Sugiyama N, De Felice M, Yeom Yl, Ohbo K, Masuko K, Obinata M, Abe K, Schöler HR, Matsui Y: Germline-specific expression of the Oct-4/green fluorescent protein (GFP) transgene in mice. Dev Growth Differ 1999, 41(6):675-684.

31. Stadtfeld M, Apostolou E, Akutsu H, Fukuda A, Follett P, Natesan S, Kono T, Shioda T, Hochedlinger $K$ : Aberrant silencing of imprinted genes on chromosome 12qF1 in mouse induced pluripotent stem cells. Nature 2010, 465(465):175-181.

32. Liu L, Luo G-Z, Yang W, Zhao X, Zheng Q, Lv Z, Li W, Wu H-J, Wang L, Wang X-J, Zhou Q: Activation of the imprinted Dlk1-Dio3 region correlates with pluripotency levels of mouse stem cells. J Biol Chem 2010, 285(25):19483-19490.

33. Watanabe K, Kamiya D, Nishiyama A, Katayama T, Nozaki S, Kawasaki H, Watanabe Y, Mizuseki K, Sasai Y: Directed differentiation of telencephalic precursors from embryonic stem cells. Nat Neurosci 2005, 8(3):288-296.

34. Abranches E, Silva M, Pradier L, Schulz H, Hummel O, Henrique D, Bekman E: Neural differentiation of embryonic stem cells in vitro: a road map to neurogenesis in the embryo. PLOS ONE 2009, 4(7):e6286.

35. Calaora V, Chazal G, Nielsen PJ, Rougon G, Moreau H: mCD24 expression in the developing mouse brain and in zones of secondary neurogenesis in the adult. Neuroscience 1996, 73(2):581-594.

36. Nieoullon V, Belvindrah R, Rougon G, Chazal G: mCD24 regulates proliferation of neuronal committed precursors in the subventricular zone. Mol Cell Neurosci 2005, 28(3):462-474.

37. Pruszak J, Sonntag K-C, Aung MH, Sanchez-Pernaute R, Isacson O: Markers and Methods for Cell Sorting of Human Embryonic Stem Cell-Derived Neural Cell Populations. Stem Cells 2007, 25(9):2257-2268.

38. Hu B-Y, Weick JP, Yu J, Ma L-X, Zhang X-Q, Thomson JA, Zhang S-C: Neural differentiation of human induced pluripotent stem cells follows developmental principles but with variable potency. Proc Natl Acad Sci USA 2010, 107(9):4335-4340.

39. Boulting GL, Kiskinis E, Croft GF, Amoroso MW, Oakley DH, Wainger BJ, Williams DJ, Kahler DJ, Yamaki M, Davidow L, Rodolfa CT, Dimos JT, Mikkilineni S, Macdermott AB, Woolf CJ, Henderson CE, Wichterle $H_{\text {, }}$ Eggan K: A functionally characterized test set of human induced pluripotent stem cells. Nature Biotechnology 2011, 29(3):279-286.

40. Kim D-S, Lee JS, Leem JW, Huh YJ, Kim JY, Kim H-S, Park I-H, Daley GQ, Hwang D-Y, Kim D-W: Robust enhancement of neural differentiation from human ES and iPS cells regardless of their innate difference in differentiation propensity. Stem Cell Rev 2010, 6(2):270-281.

41. Kondo T, Johnson SA, Yoder MC, Romand R, Hashino E: Sonic hedgehog and retinoic acid synergistically promote sensory fate specification from bone marrow-derived pluripotent stem cells. Proc Natl Acad Sci USA 2005, 102(13):4789-4794

doi:10.1186/1471-2202-12-82

Cite this article as: Koehler et al:: Extended passaging increases the efficiency of neural differentiation from induced pluripotent stem cells. BMC Neuroscience 2011 12:82.

\section{Submit your next manuscript to BioMed Central and take full advantage of:}

- Convenient online submission

- Thorough peer review

- No space constraints or color figure charges

- Immediate publication on acceptance

- Inclusion in PubMed, CAS, Scopus and Google Scholar

- Research which is freely available for redistribution

Submit your manuscript at www.biomedcentral.com/submit 